\title{
del Nido cardioplegia: Passing fad or here to stay?
}

\author{
Harold L. Lazar, MD
}

\author{
From the Division of Cardiac Surgery, Boston University School of Medicine, Boston, Mass. \\ Disclosures: Author has nothing to disclose with regard to commercial support. \\ Received for publication Sept 27, 2017; accepted for publication Sept 27, 2017; available ahead of print Oct 27, \\ 2017. \\ Address for reprints: Harold L. Lazar, MD, 80 E Concord St, Boston, MA 02118 (E-mail: Harold.1.lazar@ gmail \\ com). \\ J Thorac Cardiovasc Surg 2018;155:1009-10 \\ $0022-5223 / \$ 36.00$ \\ Copyright (C) 2017 by The American Association for Thoracic Surgery \\ https://doi.org/10.1016/j.jtcvs.2017.09.126
}

Chemical cardioplegia was developed to decrease operative morbidity and mortality by allowing surgeons to operate in a "tranquil" and "bloodless" field. Its evolution has been anything but tranquil and bloodless, however, as it has been the subject of a tremendous number of publications, research grants, and epic battles in the quest for the ideal cardioplegic technique-blood versus crystalloid, continuous versus intermittent, antegrade versus retrograde, and warm versus cold. Just when it appeared that the field of cardioplegia had truly been "arrested," along comes del Nido (DN) cardioplegia. Is this a passing fad, or is it here to stay?

DN cardioplegia is a blood-based solution with an electrolyte concentration similar to extracellular fluid with the addition of mannitol, magnesium, sodium hydrogen carbonate, and potassium chloride. It is not glucose based and relies on established glycolytic pathways for energy use. It contains lidocaine, a sodium ion-channel blocker, which results in a prolonged, hyperpolarizing arrest. DN cardioplegia, which is administered in a single dose during aortic clamping, was developed for pediatric cardiac operations, where it has achieved equivalent outcomes to other multidose cardioplegic solutions. ${ }^{1}$ The ability of DN cardioplegia to produce prolonged periods of arrest with only a single dose stimulated interest among adult cardiac surgeons, who reasoned that it could reduce crossclamp and bypass times, thereby contributing to decreased operative morbidity and mortality. Several retrospective studies involving isolated coronary artery bypass grafting and aortic valve surgery have indeed shown that DN cardioplegia may decrease crossclamp and bypass times. ${ }^{2-5}$ This has not, however, resulted in any improvement in morbidity and mortality. Although a smaller volume of cardioplegia is needed with the DN solution, it has not decreased the incidence of red blood cell transfusions, intensive care stay, or hospital stay. Although the cost of a single dose of DN solution is less than that of multidose cardioplegia, total hospitalization costs do not seem to be affected by DN cardioplegia. Intraoperative serum glucose may be lower with DN cardioplegia; however overall postoperative glycemic control does not appear to be affected. Most importantly, clinical studies performed to

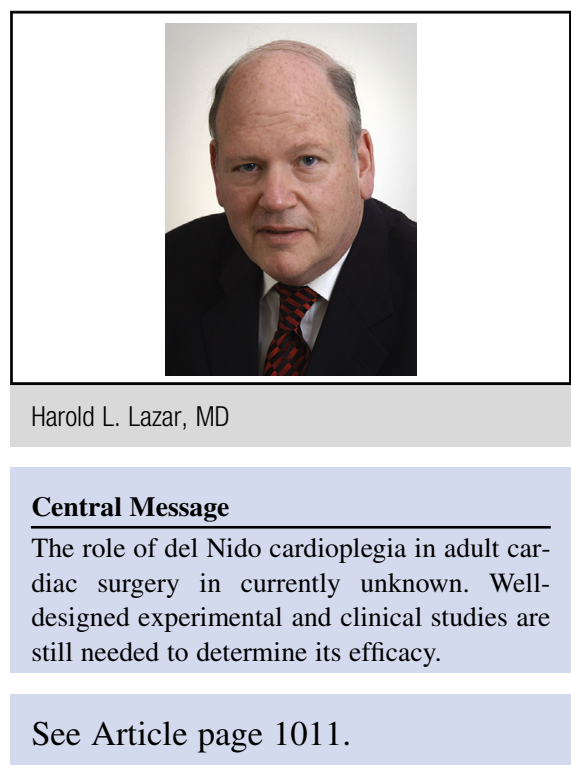

date have been retrospective and small, with a cohort of stable, elective patients with preserved left ventricular function and no right ventricular dysfunction, requiring noncomplex operative procedures. The role of DN cardioplegia in myocardial protection during adult cardiac surgery therefore remains unknown.

In this edition of the Journal, Ad and coworkers ${ }^{6}$ report their findings on the use of DN cardioplegia in a prospective, randomized study. Although Ad and coworkers ${ }^{6}$ are to be congratulated on performing a randomized trial, it unfortunately has all the shortcomings that have plagued previous studies. The sample size was small, and the crossclamp and bypass times relatively short. There was a lack of patients at high risk, especially those with unstable ischemic coronary artery disease. No assessment was made as to what, if any, significance an elevated troponin value was in the multidose group in the absence of postoperative ejection fraction and electrocardiographic changes. Previous studies have shown either no changes in troponin levels with DN cardioplegia ${ }^{2,3}$ or an actual increase in creatine kinase isoenzyme MB values. ${ }^{5}$ Although there was a decreased, albeit nonsignificant, need for inotropic support in the DN cardioplegia group, we are not told of any institutional protocol for the use of inotropic agents in cardiac surgery, and there are no data on the duration, dosage, or number of inotropes used, making this data difficult to interpret. Additional protective adjuvant techniques for myocardial protection in the patients receiving $\mathrm{DN}$, such as the use of retrograde cardioplegia, were left to the discretion of the individual surgeon and are not listed in 
the data. Although the crossclamp and bypass times were lower in the DN group, this difference was not statistically different, and resulted in no significant differences in morbidity or mortality relative to the multidose group. Furthermore, $28 \%$ of patients who received DN received an additional dose of cardioplegia. A third of the patients who received DN thus actually underwent a multidose regimen. Although the volume of DN cardioplegia administered was less than that in the multidose group, there was no difference in the number of red blood cell transfusions or in the final discharge hematocrit.

What, then, is the current role of DN cardioplegia in adult cardiac surgery? On the basis of these limited, retrospective, small size series of stable, healthy patients undergoing less complex procedures, DN cardioplegia may result in myocardial protection that is equivalent but not superior to current multidose blood cardioplegia techniques. What about those patients, however, with significant multivessel coronary artery disease with left ventricular hypertrophy, in whom cardioplegic delivery may be an issue? Or those patients with a reduced ejection fraction, who require a longer period of crossclamping for a more complicated procedure? Or those patients with pulmonary hypertension and reduced right ventricular function? Finally, when the crossclamp time exceeds 60 minutes, when should the next dose of DN cardioplegia be given, and how much?

To answer these questions, it will be necessary to proceed with lines of investigation that initially led to the development of cardioplegia in cardiac surgery-laboratory investigation and well-designed clinical trials. At present, few experimental studies exist regarding the efficacy of DN cardioplegia. In an isolated Langendorff preparation comparing single dose DN cardioplegia with multidose blood cardioplegia during 60 minutes of ischemia in "senescent rats," DN cardioplegia resulted in superior recovery of myocardial function and decreased troponin release. ${ }^{7}$ Is a "senescent rat" Langendorff model, however, equivalent to an intact porcine model of prolonged ischemic arrest on cardiopulmonary bypass? I would propose a porcine model of 3 hours of aortic clamping on cardiopulmonary bypassone group would receive an initial arresting dose of blood cardioplegia followed by intermittent doses every $20 \mathrm{mi}-$ nutes with a "terminal hot shot." The other group would receive DN cardioplegia every 60 minutes after an initial arresting dose. Parameters to be measured would include both right and left ventricular function, myocardial ATP and $\mathrm{H} 20$ content, and coronary artery vasomotor function. The use of DN cardioplegia should also be the subject of a multicenter Cardiothoracic Surgery Network trial that includes all types of cardiac procedures involving both urgent and emergency surgery. Standard protocols for the administration of both DN and multidose blood cardioplegia should be instituted and carefully followed. In addition to operative morbidity and mortality, such data as crossclamp and bypass times, need for inotropic support, intensive care unit and hospital lengths of stay, and red blood cell transfusions should also be obtained.

When flying in a jet liner, we are instructed always to keep our seat belts fastened to avoid injuries from unexpected turbulence. As we all know, cardiac surgery is filled with unexpected episodes of turbulence. Will the use of DN cardioplegia also provide optimal protection when these unexpected issues arise during surgery? Well-designed experimental and clinical studies are needed to determine whether DN cardioplegia is effective in protecting all patients undergoing all types of surgery under all conditions. Only then will we be able to determine whether DN cardioplegia is a passing fad-or it is here to stay.

\section{References}

1. Matte GS, del Nido PJ. History and use of del Nido cardioplegia solution at Boston Children's Hospital. J Exrtra Corpor Technol. 2012;44:98-103. Erratum in: J Extra Corpor Technol. 2013;45:262.

2. Timek T, Willekes C, Hulme O, Himelhoch B, Nadeau D, Borgman A, et al. Propensity matched analysis of del Nido cardioplegia in adult coronary artery bypass grafts. Ann Thorac Surg. 2016;101:2237-41.

3. Mick SL, Robich MP, Houghtaling PL, Gillinov AM, Solesz EG, Johnston DR, et al. del Nido versus Buckberg cardioplegia in adult isolated valve surgery. J Thorac Cardiovasc Surg. 2015;149:626-34; discussion 634-6.

4. Sorbella RA, Akashi H, Yerebaken H, Najjar M, Mannan A, Williams MR, et al. Myocardial protection using del Nido cardioplegia solution in adult reoperative aortic valve surgery. J Card Surg. 2014;29:445-9.

5. Yammine M, Neely RC, Loberman D, Rajab TK, Grewal A, McGurk S, et al. The use of lidocaine containing cardioplegia in surgery for adult acquired heart disease. J Card Surg. 2015;30:677-84.

6. Ad N, Holmes SD, Massimiano PS, Rongione AJ, Fornaresio LM, Fitzgerald D. The use of del Nido cardioplegia in adult cardiac surgery: a prospective randomized trial. J Thorac Cardiovasc Surg. 2018;155:1011-8.

7. Govindapillai A, Hua R, Rose R, Friesen CH, O'Blenes SB. Protecting the aged heart during cardiac surgery; use of del Nido cardioplegia provides superior functional recovery in isolated hearts. J Thorac Cardiovasc Surg. 2013;146:940-8. 